\title{
Effective population size in partially clonal plants is not predicted by the number of genetic individuals
}

\author{
Roberta Gargiulo ${ }^{1}$, Robin S. Waples ${ }^{2}$, Adri K. Grow ${ }^{3}$, Richard P. Shefferson ${ }^{4}$, Juan \\ Viruel $^{1}$, Michael F. Fay ${ }^{1}$, and Tiiu Kull ${ }^{5}$ \\ ${ }^{1}$ Royal Botanic Gardens, Kew \\ ${ }^{2}$ NOAA Fisheries \\ ${ }^{3}$ Smith College \\ ${ }^{4}$ University of Tokyo \\ ${ }^{5}$ Estonian University of Life Sciences
}

February 2, 2022

\begin{abstract}
Estimating effective population size $(\mathrm{Ne})$ is essential for theoretical and practical applications in evolutionary biology and conservation. Nevertheless, estimates of Ne in organisms with complex life-history traits remain scarce because of the challenges associated with estimation methods. Partially clonal plants capable of vegetative (clonal) growth and sexual reproduction are a common group of organisms in which the discrepancy between the apparent number of individuals (ramets) and the number of genetic individuals (genets) may be striking, and it is unclear how this discrepancy relates to Ne. In this study, we analysed two populations of the orchid Cypripedium calceolus to understand how the rate of clonal vs. sexual reproduction impacted Ne. We sampled and genotyped $>1000$ ramets at microsatellites and SNPs loci, and estimated contemporary Ne with the linkage disequilibrium method, starting from the theoretical expectation that variance in reproductive success among individuals caused by clonal reproduction and by constraints on sexual reproduction would lower Ne. We considered factors potentially affecting our estimates, including using different molecular markers and sampling strategies, and pseudoreplication in genomic datasets. The magnitude of $\mathrm{Ne} / \mathrm{Nramets}$ and $\mathrm{Ne}$ /Ngenets ratios we provide may be used as reference points for other species with similar life-history traits. Our findings demonstrate that Ne in partially clonal plants cannot be predicted based on the number of genets generated by sexual reproduction because demographic changes over time strongly influence Ne. This is especially relevant in species of conservation concern, in which population declines may not be detected by only ascertaining the number of genets.
\end{abstract}

\section{Hosted file}

db37e11b37677999eca9feebf0997728efa78f96.pdf available at https://authorea.com/users/458201/ articles/554799-effective-population-size-in-partially-clonal-plants-is-not-predictedby-the-number-of-genetic-individuals 